\title{
Pesquisa de Linfonodo-sentinela em Pacientes com Adenocarcinoma de Cólon
}

\author{
Sentinel Lymphonode Research in Patients with Colon Cancer
}

\author{
ANTÔNIOHILÁRIO ALVES FREITAS ${ }^{1}$, TARCIZO AFONSO NUNES ${ }^{2}$, ALBERTO JULIUS ALVES WAINSTEIN ${ }^{3}$, \\ ADELANIR ANTÔNIO BARROSO ${ }^{4}$, OMAR DE PAULARICARDO-FILHO $^{5}$, MARCO ANTÔNIO DIAS $^{5}$, MARCO \\ ANTÔNIO DIAS-FILHO 5 , WALDEMAR NASCIMENTO BRANDÃO PENNA 6 .
}

${ }^{1}$ Titular da Sociedade Brasileira de Coloproctologia, ${ }^{2}$ Professor UFMG, ${ }^{3}$ Pesquisador Biocâncer, ${ }^{4}$ Médico Nuclear, ${ }^{5}$ Patologista, ${ }^{6}$ Médico Residente.

FREITAS AHA; NUNES TA; WAINSTEIN AJA; BARROSO AA; RICARDO-FILHO OP; DIAS MA; DIAS-FILHO MA; PENNA WNB. Pesquisa de Linfonodo-Sentinela em Pacientes com Adenocarcinoma de Cólon. Rev bras Coloproct, 2008;28(2): 170-177.

\begin{abstract}
RESUMO: O câncer colorretal é responsável por cerca de 8.000 óbitos/ano no Brasil. Acredita-se que haja subestadiamento pósoperatório. $O$ objetivo deste trabalho é pesquisar sobre linfonodo-sentinela em pacientes com adenocarcinoma de cólon. A amostra foi composta de 18 pacientes, todos com diagnóstico de adenocarcinoma de cólon, submetidos à laparotomia com injeção dos marcadores de linfonodos na subserosa peritumoral. Resultados: a identificação intra-operatória de linfonodo-sentinela com os marcadores ocorreu em $16(88,8 \%)$ pacientes. O azul patente identificou linfonodos-sentinela em $72,2 \%$ e o fitato marcado com tecnécio em $88,8 \%$. Obtiveram-se linfocintilografias do espécime cirúrgico removido em 15 pacientes. A sensibilidade global do método foi de $66,7 \%$ e o falso negativo de 33,3\%. Depois do exame histológico com multissecção e imunoistoquímica de 11 pacientes, foi diagnosticada metástase em uma (9\%) ocorrência, sendo considerada ultra-estadiamento. Conclusões: pode-se afirmar que o procedimento é viável; o radiofármaco é mais eficaz; a linfocintilografia da peça cirúrgica é capaz de certificar a presença de captação de radiofármaco pelo linfonodo; a incidência de metástases linfonodais é, proporcionalmente, a mesma nos linfonodos-sentinela e não-sentinela; as técnicas de multissecção e imunoistoquímica contribuem para melhorar a acuidade diagnóstica de metástase linfonodal.
\end{abstract}

Descritores: Linfonodo-sentinela. Câncer. Cólon. Metástases.

\section{INTRODUÇÃO}

A incidência de câncer colorretal no Brasil é de aproximadamente 25.000 casos por ano ${ }^{1}$. O tratamento de escolha para tumores de cólon é a remoção em bloco da neoplasia e de todos os tecidos envolvidos na drenagem linfática, que é a principal via de disseminação desses tumores ${ }^{2}$. Os estadiamentos clássicos propostos têm como principal objetivo diagnosticar metástase nos linfonodos. Quando presente, o paciente é encaminhado para quimioterapia adjuvante, que aumenta o tempo livre de doença e a sobrevida ${ }^{3}$.

Sabe-se que um quarto dos pacientes operados por adenocarcinoma de cólon e sem linfonodos compro- metidos à histologia de rotina, portanto, sem indicação de terapia adjuvante, evolui para óbito em até cinco anos por recorrência local do tumor ou metástase à distância ${ }^{4,5,6}$.

O linfonodo-sentinela é considerado o primeiro a receber drenagem linfática do tumor e, desta forma, o mais provável de conter metástases ${ }^{7}$. Nele, podem-se empregar meios diagnósticos mais eficazes, como exame histológico com multissecção e imunoistoquímica, inviáveis e de alto custo para serem realizadas em todos os linfonodos dissecados ${ }^{8}$.

Diante desses fatos, este trabalho se propõe a avaliar a pesquisa de linfonodo-sentinela nos tumores do intestino grosso por meio de corante e radiofármaco no perioperatório; e utilizar além da histologia de rotina

Trabalho realizado na Universidade Federal de Minas Gerais (UFMG); Fundação Hospitalar do Estado de Minas Gerais (FHEMIG); Fundação de Amparo à Pesquisa do Estado de Minas Gerais (FAPEMIG); Instituto de Pesquisa Biocâncer. 
com hematoxilina-eosina, a multissecção de linfonodos e a imunoistoquímica com citoqueratinas, com o objetivo de melhorar o estadiamento final dos pacientes e, assim, contribuir para aumentar o índice de cura dessa afecção.

\section{PACIENTES E MÉTODOS}

Trata-se de estudo prospectivo, descritivo e analítico, sobre linfonodo-sentinela em indivíduos com adenocarcinoma de cólon, operados exclusivamente pelo autor desta pesquisa, no período de abril de 2005 a fevereiro de 2007, no Hospital Alberto Cavalcanti, que pertence à rede pública estadual de saúde da Fundação Hospitalar do Estado de Minas Gerais (FHEMIG), Belo Horizonte, Brasil.

Aprovado pelo Comitê de Ética em Pesquisa (CEP) da Fundação Hospitalar do Estado de Minas Gerais (FHEMIG), parecer $n^{\circ} 290$; e pelo Comitê de Ética em Pesquisa (COEP) da Universidade Federal de Minas Gerais (UFMG) parecer $n^{\circ} 168$.

Todos os pacientes tiveram avaliação no préoperatório, e diagnóstico histológico de adenocarcinoma de cólon com proposta de cirurgia curativa.

Os critérios de inclusão foram: pacientes com adenocarcinoma de cólon e adesão ao termo de consentimento. Os de exclusão foram: evidência de invasão de órgão adjacente ou metástase distante; tumor de origem não identificada nos últimos cinco anos; cirurgia prévia na região que possa alterar a drenagem linfática local; gravidez; recusa do paciente.

A marcação dos linfonodos foi realizada da seguinte forma: a) injeção intra-operatória com seringa na subserosa em quatro pontos cardeais adjacentes

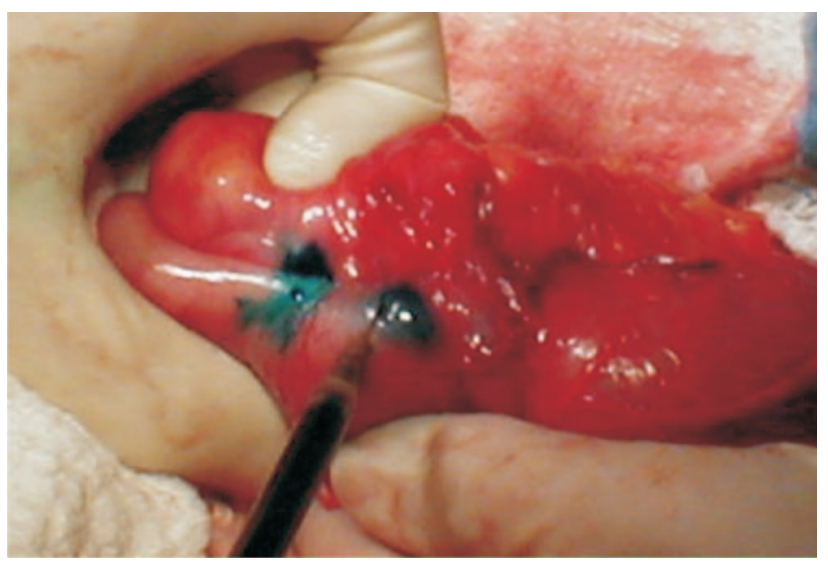

Figura 1 - Fotografia digital da injeção do marcador de linfonodos peri-tumoral. ao tumor de corante azul patente: volume de $1 \mathrm{ml}$, concentração a 1\%, fabricado pela Citopharma Ltda (Belo Horizonte - M.G.) (figura 1). b) injeção de fármaco radioativo (Fitato ${ }^{99 \mathrm{~m}}$ Tecnécio) na dose de $1,2 \mathrm{mci}$ em volume de $0,8 \mathrm{ml}$, liofilizado, estéril, apirógeno, produzido e distribuído pelo Instituto de Pesquisa de Energia Nuclear (IPEN) (São Paulo - S.P.), do mesmo modo descrito anteriormente para o corante.

A identificação dos linfonodos-sentinela foi: a) visual depois de 5-10 minutos da injeção do azul patente, com os primeiros linfonodos corados marcados com fio prolene; b) utilizada sonda captadora de raios gama (Eurorad ${ }^{\circledR}$ ) no intra-operatório para localizar linfonodos no mesocólon depois de 20-30 minutos da injeção do radiofármaco, que são marcados com fio seda (figura 2); c) adicionalmente leva-se o espécime cirúrgico fresco removido até a clínica de medicina nuclear para obter imagem cintilográfica (figura 3 ).

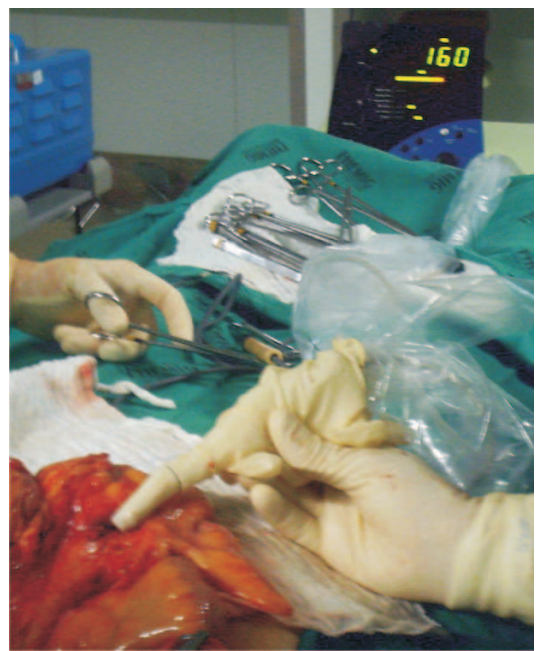

Figura 2 - Fotografia digital da identificação peroperatória dos linfonodos captantes por meio do gama probe.

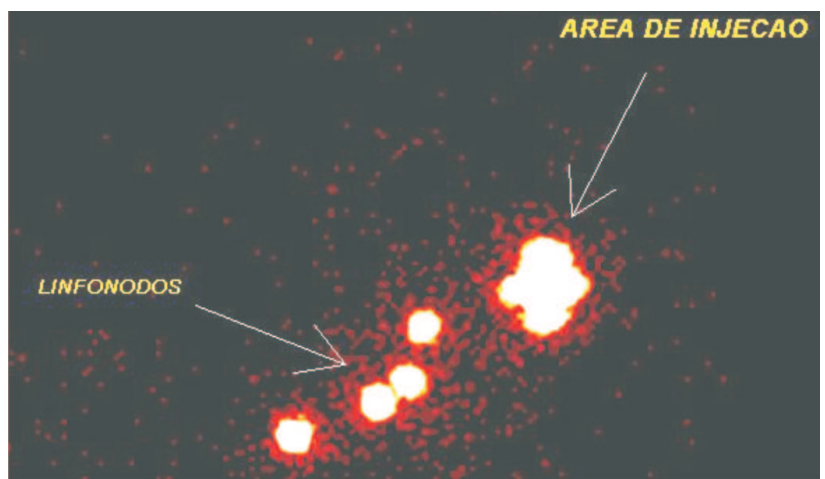

Figura 3 - Linfocintilografia da peça cirúrgica com imagem da área de injeção do radiofármaco e migração do marcador para os linfonodos-sentinela. 
Finalmente os linfonodos considerados sentinela são dissecados, e enviados com a peça cirúrgica ao serviço de anatomia patológica para realização de histologia. A análise inicial dos linfonodos-sentinela é feita pelo método de rotina, corado com hematoxilina-eosina. Em pacientes sem metástases, envia-se o linfonodo-sentinela para exames adicionais com multissecção onde são feitos múltiplos cortes, e imunoistoquímica com citoqueratinas.

O tamanho dos tumores variou de 2 a $7 \mathrm{~cm}$, com média de 4,5 cm $(\mathrm{DP}=1,4)$ e a localização no cólon foi a seguinte: direito, oito $(44,4 \%)$; esquerdo, quatro $(22,2 \%)$; e sigmóide, seis $(33,3 \%)$.

Os pacientes foram estadiados segundo a classificação patológica do tumor-nodo-metástase (TNM), preconizada por Hensen e Hutter ${ }^{9}$.

\section{RESULTADOS}

Identificação dos linfonodos pelo azul patente e radiofármaco

Tabela 1 - Resultados.
Nas peças cirúrgicas dos 18 participantes, foram isolados 238 linfonodos, média de 13,2 $(\mathrm{DP}=5,1)$ por paciente. Foram identificados 46 linfonodos-sentinela no intra-operatório por meio do corante e radiofármaco em $16(88,8 \%)$, com média de $2,8(\mathrm{DP}=0,9)$ por paciente. $\mathrm{O}$ azul patente identificou linfonodo-sentinela em 13 $(72,2 \%)$ indivíduos e o radiofármaco em 16 $(88,8 \%)$ casos. Em dois pacientes não foram identificados linfonodos-sentinela por nenhum dos marcadores.

Não foram identificados linfonodos corados ou captantes fora da área de ressecção, e por isso a extensão da operação, que foi oncológica e radical, não teve de ser ampliada. Dois apresentaram urina azul após injeção do corante, que desapareceu em aproximadamente quatro horas, não acarretando qualquer conseqüência posterior. Não houve qualquer outro evento adverso decorrente da pesquisa de linfonodo-sentinela (tabela 1).

\begin{tabular}{|c|c|c|c|c|c|c|c|c|c|c|c|}
\hline Paciente & $\begin{array}{l}\text { Tamanho } \\
\text { doTumor } \\
\text { (cm) }\end{array}$ & $\begin{array}{l}\text { Local } \\
\text { Tumor } \\
\text { Cólon }\end{array}$ & $\begin{array}{l}\text { LNS } \\
\text { Azul }\end{array}$ & $\begin{array}{l}\text { LNS } \\
\text { Radio }\end{array}$ & $\begin{array}{c}\text { Imagen } \\
\mathbf{L N}\end{array}$ & $\begin{array}{c}\text { Total } \\
\text { LN }\end{array}$ & $\begin{array}{c}\text { Metas } \\
\text { em LNS } \\
\text { (H-E) }\end{array}$ & $\begin{array}{c}\text { Metas } \\
\text { em LNNS } \\
\text { (H-E) }\end{array}$ & $\begin{array}{c}\text { Estadio } \\
\text { TNM }\end{array}$ & MS & IQ \\
\hline 1-EPR & 6,5 & direito & 01 & 04 & Sim & 12 & 00/04 & $00 / 08$ & II & Negativa & Negativa \\
\hline 2-MJC & 5 & sigmóide & 00 & 00 & Sim & 14 & $00 / 00$ & $01 / 14$ & III & NR & $\mathrm{NR}$ \\
\hline 3-GFL & 5,5 & direito & 01 & 02 & $\mathrm{Nr}$ & 12 & $00 / 02$ & $00 / 10$ & II & Negativa & Negativa \\
\hline 4-GAS & 7 & direito & 00 & 01 & $\mathrm{Nr}$ & 14 & 01/01 & $02 / 13$ & III & NR & $\mathrm{NR}$ \\
\hline 5-MAR & 4 & direito & 03 & 01 & Sim & 09 & $03 / 03$ & $00 / 06$ & III & NR & $\mathrm{NR}$ \\
\hline 6-VLM & 5 & esquerdo & 04 & 04 & Sim & 12 & $00 / 04$ & $00 / 08$ & II & Positiva & Positiva \\
\hline 7-MJD & 3,5 & sigmóide & 00 & 01 & Sim & 12 & $01 / 01$ & $08 / 11$ & III & NR & $\mathrm{NR}$ \\
\hline 8-PAS & 3,5 & esquerdo & 01 & 01 & Não & 06 & $00 / 01$ & $00 / 05$ & II & Negativa & Negativa \\
\hline 9-RCSJ & 4 & sigmóide & 04 & 04 & Sim & 14 & $00 / 04$ & $01 / 10$ & III & NR & $\mathrm{NR}$ \\
\hline 10-ANF & 4 & esquerdo & 03 & 03 & Sim & 13 & $00 / 03$ & $00 / 10$ & II & Negativa & Negativa \\
\hline 11-ALM & 4 & sigmóide & 02 & 02 & Sim & 15 & $00 / 03$ & $00 / 12$ & II & Negativa & Negativa \\
\hline 12-MLCS & 6 & direito & 02 & 04 & Sim & 15 & $01 / 04$ & $05 / 11$ & III & NR & $\mathrm{NR}$ \\
\hline 13-COM & 2 & direito & 04 & 04 & Sim & 24 & $00 / 04$ & $00 / 20$ & I & Negativa & Negativa \\
\hline 14-ROG & 2 & esquerdo & 00 & 00 & Sim & 07 & $00 / 00$ & $00 / 07$ & I & Negativa & Negativa \\
\hline 15-SAS & 4 & direito & 01 & 04 & Sim & 24 & $00 / 04$ & $00 / 20$ & II & Negativa & Negativa \\
\hline 16-JRA & 4 & sigmóide & 02 & 02 & Sim & 04 & $00 / 02$ & $00 / 02$ & II & Negativa & Negativa \\
\hline $17-\mathrm{ACC}$ & 6 & sigmóide & 00 & 02 & $\mathrm{Nr}$ & 16 & $00 / 02$ & $07 / 14$ & III & NR & $\mathrm{NR}$ \\
\hline 18-MVA & 6 & direito & 01 & 04 & Sim & 15 & $00 / 04$ & $00 / 11$ & II & Negativa & Negativa \\
\hline Total & & & 29 & 43 & & 238 & $06 / 46$ & $24 / 192$ & & & \\
\hline
\end{tabular}

Legenda:

$L N=$ linfonodo, $L N S=$ linfonodo-sentinela, $L N N S=$ linfonodo não-sentinela $; H-E=$ hematoxilina-eosina $($ exame histológico de rotina), $M S$ $=$ multissecção de linfonodos, $I Q=$ imunoistoquímica de linfonodos, $N R=$ náo realizado, Azul = captante de corante, Radio $=$ captante de radiofármaco. 


\section{Linfocintilografia}

Foram obtidas imagens das peças cirúrgicas de 15 pacientes, o que não foi possível nos outros três por problemas logísticos. Nos dois casos em que os linfonodos-sentinela não foram identificados no intraoperatório, também não foram detectadas imagens de linfonodos por cintilografia.

Treze pacientes tiveram linfonodos-sentinela identificados no intra-operatório e também foram realizadas imagens na gama-câmara. Em 12 (92,3\%) destes apareceram linfonodos na imagem e em apenas um caso $(7,7 \%)$ a linfocintilografia não mostrou imagem de linfonodo.

\section{Marcadores de linfonodos e histologia}

Entre os 46 linfonodos-sentinela identificados pelos marcadores, o exame histológico de rotina com hematoxilina-eosina diagnosticou metástases em seis $(13,0 \%)$, e nos outros $40(87,0 \%)$ não foram encontrados sinais de metástases. Dos 192 linfonodos não-sentinela, $24(12,5 \%)$ continham metástases e $168(87,5 \%)$ estavam livres de metástases (tabela 2).
Considerando o estudo de validade, nos 16 pacientes cujos marcadores identificaram linfonodos-sentinela, o exame histológico de rotina diagnosticou metástase em seis (37,5\%). Em quatro (66,7\%) deste grupo, as metástases encontravam-se nos linfonodossentinela, e em dois $(33,3 \%)$ em linfonodos não-sentinela, sendo que este último índice representa o falsonegativo. Nos outros 10 pacientes, apesar de terem sido identificados linfonodos-sentinela, não foram diagnosticadas metástases pela histologia de rotina com hematoxilina-eosina (tabela 3 ).

Analisando a concordância entre os marcadores neste grupo de sete pacientes cujos linfonodos continham metástases ao exame histológico de rotina com hematoxilina-eosina, seis $(85,7 \%)$ tiveram linfonodos captantes de radiofármaco e três $(42,8 \%)$ tiveram linfonodos corados pelo azul (tabela 4).

\section{Estadiamento dos pacientes}

O exame histológico de rotina não diagnosticou metástase em $11(61,1 \%)$ dos 18 pacientes,

Tabela 2 - Resultado da histologia de rotina com hematoxilina-eosina dos linfonodos nas 18 peças cirúrgicas $(n=238)$.

\begin{tabular}{lrrr}
\hline Linfonodos & Metástase (+) & Metástase (-) & Total \\
\hline Sentinela & $6(13,0 \%)$ & $40(87,0 \%)$ & $46(100,0 \%)$ \\
Não sentinela & $24(12,5 \%)$ & $168(87,5 \%)$ & $192(100,0 \%)$ \\
Total & 30 & 208 & 238 \\
\hline
\end{tabular}

$(+)=$ positivo para metástase.

$(-)$ = negativo para metástase.

$p=0,88$.

Tabela 3 - Histologia nos pacientes com câncer de cólon, nos quais foram identificados linfonodos-sentinela $(n=16)$.

\begin{tabular}{|c|c|c|c|}
\hline Linfonodos & Com Metástase & Sem Metástase & Total \\
\hline$\overline{\text { Sentinela }}$ & 4 & 0 & 4 \\
\hline Não-sentinela & 2 & 10 & 12 \\
\hline Total & 6 & 10 & 16 \\
\hline
\end{tabular}

Sensibilidade $=66,7 \% \quad($ IC 95\% - 24,1\% a 94,0\% $)$.

Especificidade $=100 \% \quad($ IC $95 \%-65,5 \%$ a $100 \%)$.

Valor Preditivo Positivo $=100 \%$ (IC 95\% - 39,6\% a 100\%).

Valor Preditivo Negativo $=83,3 \%($ IC $95 \%-50,9 \%$ a 97,1\%).

Acurácia $=87,5 \%$ (IC 95\% - 60,4\% a 97,8\%).

IC-intervalo de confiança. 
Tabela 4 - Correlação entre os marcadores nos pacientes com metástases nos linfonodos $(n=7)$.

\begin{tabular}{lccc}
\hline Pacientes & Azul (+) & Azul (-) & Total \\
\hline Fitato $(+)$ & 3 & 3 & 6 \\
Fitato $(-)$ & 0 & 1 & 1 \\
Total & 3 & 4 & 7 \\
\hline
\end{tabular}

$(+)=$ captante de marcador

$(-)=$ não captante de marcador.

Índice Kappa $=22 \%$.

portanto, eles foram considerados estádio N0. Nos outros sete $(38,9 \%)$, foram diagnosticadas metástases, sendo classificados no estádio N1 ou N2. Dessa forma, o estadiamento (TNM) inicial da amostra foi:

$$
\begin{aligned}
& \text { Estádio I }=02 \text { pacientes }(11,1 \%) ; \\
& \text { Estádio II }=09 \text { pacientes }(50,0 \%) \\
& \text { Estádio III }=07 \text { pacientes }(38,9 \%) .
\end{aligned}
$$

No grupo dos 11 pacientes sem metástases pela histologia de rotina (estádios I ou II), os exames por multissecção e imunoistoquímica diagnosticaram metástase em apenas um $(9,0 \%)$. O estadiamento (TNM) final, então, foi:

$$
\begin{aligned}
& \text { Estádio I }=02 \text { pacientes }(11,1 \%) \\
& \text { Estádio II }=08 \text { pacientes }(44,45 \%) \\
& \text { Estádio III }=08 \text { pacientes }(44,45 \%)
\end{aligned}
$$

\section{DISCUSSÃO}

\section{Identificação dos linfonodos}

O mapeamento linfático do câncer de cólon é realizado pela maioria dos autores com injeção subserosa dos marcadores de linfonodos (corantes e/ ou fármacos radioativos) no intra-operatório, método utilizado no presente estudo.

Neste estudo, empregou-se a técnica combinada (corante e radiofármaco), pois não há consenso de qual seria o melhor marcador ${ }^{10,11,12}$. Apesar de mais trabalhosa, ainda foi acrescida a linfocintilografia, na busca por melhores resultados. $O$ índice de sucesso na identificação de linfonodos-sentinela em adenocarcinomas de cólon foi de $88,8 \%$. Isto mostra a viabilidade técnica, já que mesmo no projeto piloto com os quatro primeiros casos não houve dificuldade com o método empregado, fato verificado por Paramo et al. ${ }^{13}$, que calcularam a curva de aprendizado, sendo necessários cinco pacientes.
Basílio e Fonseca ${ }^{14}$, no Rio de Janeiro, utilizaram o corante e identificaram linfonodos-sentinela em todos os 31 pacientes estudados.

Saha et al. ${ }^{15}$, equipe com mais experiência mundial nesse método, afirmaram que a utilização da técnica combinada, apesar de mais trabalhosa, pode aumentar a detecção de linfonodos-sentinela em pacientes com tumores de cólon. Patten et al. ${ }^{16}$ discordam, uma vez que em sua avaliação a sensibilidade permaneceu inalterada quando se utilizou corante associado ao fármaco radioativo.

Doekhie et al. ${ }^{17}$ revisaram 25 estudos, sendo que destes, 19 utilizaram o corante azul como marcador, quatro técnicas combinadas de azul mais radiofármaco, e em apenas dois o fármaco radioativo isoladamente, com $89 \%$ de sucesso na identificação dos linfonodos-sentinela. Em recentes revisões de trabalhos relevantes, levando-se em consideração diferentes técnicas, a identificação de linfonodo-sentinela variou de 71 a $100 \%{ }^{18,19}$.

\section{Marcadores}

Neste estudo, o desempenho do fitato radioativo foi melhor que o do corante azul patente para identificação de linfonodos-sentinela. Todos aqueles com linfonodos metastáticos e que tiveram linfonodos corados de azul, foram também captantes de radiofármaco, ou seja, o fármaco radioativo agregou valor na identificação de linfonodo-sentinela.

Hipótese que poderia explicar tal fato seria a desvantagem da farmacocinética do corante azul com rápida passagem pelo linfonodo e metabolização instantânea pelo sistema imunológico. Falhas técnicas também podem ocorrer na injeção dos marcadores ${ }^{20,21,22}$.

\section{Linfocintilografia}

Foram obtidas imagens das peças cirúrgicas levadas à gama-câmara de 15 pacientes envolvidos no trabalho, que serviram para certificação da captação pelos linfonodos do radiofármaco injetado no intra-operatório. Nos dois $(12,2 \%)$ pacientes em que os linfonodos-sentinela não foram identificados pelos métodos intra-operatórios, também não foi detectada imagem de linfonodo no mesocólon.

Poucos trabalhos foram publicados sobre este tema, provavelmente devido ao alto custo, que seria o fator limitante principal, já que o valor de cada linfocintilografia é de aproximadamente 600 dólares ou 1.200 reais. Merrie et al. ${ }^{21}$ fizeram linfocintilografia 
objetivando localizar os linfonodos radiocaptantes no mesocólon do espécime cirúrgico, e obtiveram resultados favoráveis.

\section{Captação dos marcadores e histologia}

O número de linfonodos obtidos do espécime cirúrgico é de grande importância para estadiamento, tratamento e prognóstico dos pacientes operados de adenocarcinoma de cólon. Nos 18 casos estudados, foi encontrada média de 13,2 linfonodos por paciente, que de acordo com a literatura é suficiente para estadiamento confiável ${ }^{23,24,25}$. Não existiu correlação significativa $(\mathrm{p}=0,88)$ entre linfonodo-sentinela e a presença de metástase.

\section{Falso-negativo e sensibilidade}

$\mathrm{O}$ alto índice de falso-negativo $(33,3 \%)$ é significativo, mas não interfere na terapêutica, já que a linfadenectomia cirúrgica radical é mantida nas operações de adenocarcinomas de cólon, independentemente da presença ou ausência de metástases nos linfonodos regionais. De acordo com os trabalhos de revisão, a taxa global média de falso-negativo é de $33 \%$, variando de 0 a $63 \%$. Quando observados somente os trabalhos que utilizaram o método combinado (corante e radiofármaco), como nesta avaliação, a taxa média foi de $28 \%$, variando de 0 a $50 \%$ 19, 27, 29 .

De acordo com o estudo de validade deste trabalho, a sensibilidade foi $66,7 \%$, enquanto na literatura varia de 40 a $100 \%{ }^{17,26,28}$.

Há um consenso que quanto mais avançado for o tumor, mais alto deverá ser o índice de falsonegativo e menor a sensibilidade. Como este trabalho foi desenvolvido em hospital público e os pacientes geralmente têm dificuldade para realização de propedêutica, esta seria uma provável causa para justificar os valores encontrados. Ressalta-se que 38,9\% dos pacientes já se encontravam no estádio III (linfonodos com metástases) depois da histologia de rotina com hematoxilina-eosina.

\section{Estadiamento}

De acordo com o padrão de exame histológico de rotina, o tecido é examinado por meio de secção simples do linfonodo. Estima-se que o material levado a exame microscópico corado pela hematoxilina-eosina corresponda a menos de $1 \%$ do tecido do linfonodo, podendo ser motivo potencial de erro ${ }^{30,31}$.
Neste estudo, em um (9\%) paciente foi encontrada micrometástase em linfonodo-sentinela depois da realização de exame histológico complementar, utilizando técnicas de multissecção dos linfonodos e de imunoistoquímica. Segundo Cohen et al. ${ }^{32}$, poderia representar um caso de provável subestadiamento, cujo perfil se beneficiaria da pesquisa de linfonodo-sentinela por ter o diagnóstico de sua doença ainda micrometastática, quando as chances de proporcionar a cura com uma quimioterapia adjuvante seriam maiores por atacar o tumor em fase inicial.

O ultra-estadiamento por meio de multissecção e imunoistoquímica foi 4-47\% na revisão de Tuech et al. ${ }^{26}$; de $37,5 \%$ segundo Codignola et al. ${ }^{33}$; e variou de $0-50 \%$ na revisão de Doekhie et al. ${ }^{17}$.

O'Brien et al. ${ }^{34}$ objetivaram melhorar o estadiamento dos linfonodos utilizando método imunoistoquímico para diagnóstico de micrometástases (depósitos isolados ou grupos de células tumorais, de acordo com Huvus et al. ${ }^{35}$ ). Verificou-se que em cinco estudos os resultados foram favoráveis, e em nove não foram observados efeitos na evolução dos pacientes ${ }^{26}$. Wong et al. ${ }^{36}$ verificaram que nos linfonodossentinela há mais presença de metástases ocultas que nos não-sentinela, mas como utilizaram metodologias diferentes para exame dos linfonodos, tiveram a validade de seus achados muito questionada.

Portanto, são necessários mais trabalhos confiáveis e com grandes amostras para avaliação do real papel das micrometástases na evolução, na sobrevida e também a aplicabilidade prática, para que possa ser respondido se os pacientes operados de adenocarcinoma de cólon deveriam ou não receber terapia adjuvante nessas situações especiais ${ }^{37,38}$.

\section{CONCLUSÕES}

Diante do resultado da pesquisa de linfonodosentinela por meio de corante e radiofármaco no perioperatório de pacientes com adenocarcinoma de cólon, pode-se afirmar que: o procedimento é viável, seguro e com índice de sucesso elevado; a associação dos marcadores é benéfica, porém o radiofármaco é mais eficaz; a linfocintilografia da peça cirúrgica é capaz de certificar a presença de captação de radiofármaco pelo linfonodo; a incidência de metástases linfonodais é, proporcionalmente, a mesma nos linfonodos-sentinela e não-sentinela, e falso-negativo de $33 \%$, portanto a linfadenectomia radical deve ser 
Rev bras Coloproct Abril/Junho, 2008
Pesquisa de Linfonodo-Sentinela em Pacientes

com Adenocarcinoma de Cólon

Antônio Hilário Alves Freitas e Cols.
Vol. 28 usada em todos os casos; as técnicas de multissecção e imunoistoquímica alteraram o estadiamento em um dos pacientes do estudo, contribuindo para melhorar a acuidade diagnóstica de metástase linfonodal.

\begin{abstract}
The colorectal cancer is responsible for $\mathbf{8 . 0 0 0}$ deaths a year in Brazil. It is believed that there is post operative sub staged. The objective of this study is to research on the sentinel lymph node in patients with colon cancer. The sample was composed by 18 patients, all of them with diagnose of cancer, undertaken to laparotomy with injection of the markers of lymph nodes in the subserosa peritumoral. Results: intraoperative identification sentinel lymph nodes with the markers occurred in 16 $(88,8 \%)$ patients. The patent blue dye identified sentinel lymph nodes in $13(72,2 \%)$ and the radioisotopic in $16(88,8 \%)$. Lymphoscintigraphy of surgical specimen were obtained from 15 patients. The global sensitivity of this method was of $66,7 \%$ and the false-negative of $33,3 \%$. After the histological examination with multilevel section and immunohistochemical in 11 patients, one $(9 \%)$ case of micrometastase was diagnosed being considered ultrastaging. Conclusions: It can be said that the procedure is viable; the radioisotope is more effective; the lymphoscintigraphy of the surgical specimen is capable of certifying the presence of absorption of the radioisotope by the lymph node; the incidence of lymph node metastases is, proportionally, the same as the one of the sentinel and non sentinel nodes; the techniques of the multilevel section and immunohistochemical contribute to improve the activity of the lymph node metastases diagnose.
\end{abstract}

Key words: Sentinel lymph node. Cancer. Colon. Metastases.

\section{REFERENCIAS}

1. Instituto Nacional do Câncer (INCA), 2006. Disponível em: http://www.inca.gov.br.

2. Gordon PH, Nivatvongs S. Principles and practice of surgery for the colon, rectum, and anus, 2th ed. St Louis, Missouri: Quality medical Publishing, Inc., 1999.

3. Wolmark N, Rockette H, Mamounas EP. The relative efficacy of 5-FU + leucovorin (FU-LV), 5-FU + levamisole (FU-Lev) and 5$\mathrm{FU}+$ leucovorin + levamisole (FU-LV-Lev) in patients with Dukes B and C carcinoma of the colon: first report of NSABP C04 [abstract 460]. Proc Am Soc Clin Oncol, 1996; 15:205.

4. Bilchick AJ, Nora DT. Lymphatic mapping of nodal micrometastasis in colon câncer: putting the cart before the horse? Ann Surg Oncol, 2002; 9:529-531.

5. Bertoglio S, Sandrucci S, Percivale P, Goss M, Gipponi M, Moresco L, Mussa B, Mussa A. Prognostic value of sentinel lymph node biopsy in the pathologic staging of colorectal cancer patients. J Surg Oncol, 2004; 85:166-170.

6. Saha S, Seghal R, Patel M, Doan K, Dan A, Bilchick A, Beutler T, Wiese D, Bassily N, Yee C. A multicenter trial of sentinel lymph node mapping in colorectal cancer: prognostic implications for nodal staging and recurrence. Am J Surg, 2006; 191:305-310.

7. Morton DL, Wen DR, Wong JH, Economou JS, Cagle LA, Storm FK, Foshag LJ, Cochran AJ. Technical details of intraoperative lymphatic mapping for early stage melanoma. Arch Surg, 1992; 127:392-399.

8. Wainstein AJA, Barroso AA, Belfort AF. Significado do linfonodo-sentinela no câncer do aparelho digestivo. Tópicos em gastroenterologia 14. Editora Medsi, Rio de Janeiro, RJ, 2004.
9. Hensen DE, Hutter RV. American Joint Commettee for Cancer. In: Behars $\mathrm{OH}$, Myers MH. Manual for staging of cancer. Philadelphia: JB Lippincot, 1988, pp 75-80.

10. Sprung J, Tully MJ, Ziser A. Anaphilatic reactions to isosulfan blue dye during sentinel node lymphadenectomy for breast cancer. Anesth Analg, 2003; 96:1051-1053.

11. Nastro P, Sodo M, Dodaro CA, Gargiulo S, Acampa W, Bracale $\mathrm{U}$, Renda A. Intraoperative radiochromoguided mapping of sentinel lymph node in colon câncer. Tumori, Milão, v.88: p.352-353, 2002.

12. Saha S, Dan AG, Berman B, Wiese D, Schochet E, Barber K, Choudhri S, Kaushal S, Ganatra B, Desai D, Nagaraju M, Mannam S. Lymphazurin $1 \%$ versus ${ }^{99 \mathrm{~m}} \mathrm{Tc}$ sulfur colloid for lymphatic mapping in colorectal tumors: a comparative analysis. Ann Surg Oncol, 2004; 11:21-26.

13. Paramo JC, Summerall J, Poppiti R, Mesko TW. Validation of sentinel node mapping in patients with colon cancer. Ann Surg Oncol, 2002; 9:550-554.

14. Basílio P, Fonseca LMB. Detecção de linfonodo-sentinela no câncer colorretal. Arq Gastroenterol, 2006; 43:163-167.

15. Saha S, Dan AG, Beutler T, Wiese D, Badin J, Branigan T, Peter Ng, Bassily N, David D. Sentinel lymph node mapping technique in colon cancer. Semin Oncol, 2004; 31:374-381.

16. Patten LC, Berger DH, Bigas MR, Mansfield P, Delpassand E, Cleary KR, Fagan SP, Curley SA, Hunt KK, Feig BW. A prospective evaluation of radiocolloid and immunohistochemical staining in colon carcinoma lymphatic mapping. Cancer, 2004; 100:2104-2109.

17. Doekhie FS, Peeters KCMJ, Kuppen PJK, Mesker WE, Tanke HJ, Morreau H, Velde CHJ, Tollenaar RAEM. The feasibility and reliability of sentinel node mapping in colorectal cancer. Eur J Soc Oncol, 2005; 31:854-862. 
18. De Haas RJ, Wicherts DA, Hobbelink MGG, Rinkes IHMB, Schipper MEI, van der Zee J, Hillegersberg R. Sentinel lymph node mapping in colon cancer: current status. Ann Surg Oncol, 2007; 14:1070-1080.

19. Dess Guetz G, Uzzan B, Nicolas P, Cucherat M, Mestier P, Morere JF, Breau JL, Perret G. Is sentinel lymph node mapping in colorectal cancer a future prognostic factor? A meta-analysis. World J Surg, 2007; 31:1304-1312.

20. Feig BW, Curley S, Lucci A, Hunt KK, Vauthey JN, Mansfield PF, Cleary K, Hamilton S, Ellis V, Brame M, Berger DH. A caution regarding lymphatic mapping in patients with colon cancer. Am J Surg, 2001; 182:707-712.

21. Merrie AEH, van Rij A, Phillips L, Rossaak J, Yun K, McCall JL. Diagnostic use of sentinel node in colon cancer. Dis Colon Rectum, 2001; 44:410-417.

22. Viehl CT, Hamel CT, Marti WR, Guller U, Eisner L, Stammberger U, Terracciano L, Spichtin HP, Harder F, Zuber M. Identification of sentinel lymph nodes in colon cancer depends on the amount of dye injected relative to tumor size. World J Surg, 2003, 27:1285-1290.

23. Greene FL. American Joint Commettee on Cancer: cancer staging handbook, TNM classification of malignant tumors. New York: Springer-Verlag, 2002.

24. Benson AB, Schrag D, Somerfield MR, Cohen AM, Figueredo AT, Flynn PJ, Krzyzanowska MK, Maroun J, McAllister P, Cutsem EV, Brouwers, Charette M, Haller DG. American society of clinical oncology recommendations on adjuvant chemotherapy for stage II colon cancer. J Clin Oncol, 2004; 22:3408-3419.

25. Rossi BM, Bepu Jr P, Ferreira FO, Santos EMM, Aguiar Jr S, Nakagawa WT, Lopes A. Number of dissected lymph nodes in colorectal cancer patients submitted to radical surgery: the quality of oncology treatment. Applied Cancer Research, 2006; 26:27-33.

26. Tuech JJ, Pessaux P, Regenet N, Bergamaschi R, Colson A. Sentinel lymph node mapping in colon cancer. Surg Endosc, 2004; 18:1721-1729.

27. Tiffet O, Kaczmarek D, Chambonierre MR, Guillan T, Baccot S, Prevot N, Bageacu S, Bourgeois E, Cassagnau E, Lehur PA, Dubois F. Combining radioisotopic and blue-dye technique does not improve the false-negative rate in sentinel lymph node mapping for colorectal cancer. Dis Col Rectum, 2007; 50:962-970.

28. Kitagawa Y, Watanabe M, Hasegawa H, Yamamoto S, Fujii H, Yamamoto K, Matsuda J, Mukai M, Kubo A, Kitajima M.
Sentinel node mapping for colorectal cancer with radioactive tracer. Dis Colon Rectum, 2002; 45:1476-1480.

29. Joosten JJA, Strobbe LJA, Wauters CAP, Pruszczynski M, Wobbes T, Ruers TJM. Intraoperative lymphatic mapping and the sentinel node concept in colorectal carcinoma. Br J Surg, 1999; 86:482-486.

30. Paraf F, Sabourin JC. Optimal lymph node number and occult lymph node metastases in colorectal cancer: the pathologists view. Gastroenterol Clin Biol, 2000; 24:423-459.

31. Tschmelitsch J, Klimstra DS, Cohen AM. Lymph node micrometastases do not predict relapse in stage II colon cancer. Ann Surg Oncol, 2000; 7:601-608.

32. Cohen AM, Tremiterra S, Candeh F. Adjuvant therapy for colorectal cancer. Curr Prob Cancer, 1998; 22:5-65.

33. Codignola C, Zorzi F, Mutti S, Rizzi A, Padelecchia E, Morandi GB. Is there any role for sentinel node mapping in colorectal cancer staging? Personal experience and review of the literature. Jpn J Clin Oncol, 2005; 35:645-650.

34. O'Brien MJ. Immunocytochemical localization of carcinoembryogenic antigen in benign and malignant colorectal tissues. Assesment of diagnostic value. Am J Clin Pathol, 1981; 75:283-290.

35. Huvus AG, Hutter RV, Berg JW. Significance of axillary macrometastases and micrometastases in mammary cancer. Ann Surg, 1971; 173:44-46.

36. Wong JH, Johnson DS, Namike P, Tauchi NP. Validation of ex vivo lymphatic mapping in hematoxylin-eosin node-negative carcinoma of the colon and rectum. Ann Surg Oncol, 2004; 11:772-777.

37. Bembenek AE, Schineider U, Gretschel S, Fisher J. Detection of lymph node micrometastases and isolated tumor cells in sentinel and nonsentinel lymph nodes of colon cancer patients. World J Surg, 2005; 29:1172-1175.

38. Bendavid Y, Latulippe F, Younan RJ, Leclerc YE, Dube S, Heyen F, Morin M, Girard R, Bastien E, Ferreira J, Cerino M, Dube P. Phase I study on sentinel lymph node mapping in colon cancer: a preliminary report. J Surg Oncol, 2002; 79:81-84.

Endereço para correspondência: ANTÔNIO HILÁRIO ALVES FREITAS

Rua Paracatu, 838 / 206 - Barro Preto

30180.090 - Belo Horizonte - Minas Gerais

Telefone e FAX: 31-3337.9988 Celular: 31-9956.1363

E-mail: hilariofreitas@ig.com.br 